Original Research Paper

\title{
Microstrip Bandpass Filter with Wide Stopband and High Out-of-Band Rejection
}

\author{
${ }^{1}$ Mohamad Farhat, ${ }^{2}$ Bal Virdee and ${ }^{1}$ Nader H. Ghareeb \\ ${ }^{I}$ School of Engineering, Australian College of Kuwait, Kuwait \\ ${ }^{2}$ School of Computing and Digital Media, London Metropolitan University, UK
}

\author{
Article history \\ Received: 17-11-2019 \\ Revised: 20-12-2019 \\ Accepted: 27-12-2019 \\ Corresponding Author: \\ Mohamad Farhat \\ School of Engineering, \\ Australian College of Kuwait, \\ Kuwait \\ Email: m.farhat@ack.edu.kw
}

\begin{abstract}
This paper presents a compact microstrip bandpass filter exhibiting a wide stopband, high selectivity and easily tunable passband. The filter comprises of asymmetric resonator structures, which are interconnected by an inter-digital capacitor to enable the realization of a wide bandwidth with high rejection level. High selectivity and easily tunable passband are obtained by optimizing the parameters of the interdigital capacitor. The filter has high out-of-band rejection $30 \mathrm{~dB}$, less than $0.6 \mathrm{~dB}$ of insertion-loss, up to $5.5 \mathrm{GHz}$ spurii free and more than 15 $\mathrm{dB}$ of return-loss. Full-wave electromagnetic simulator $\mathrm{ADS}^{\mathrm{TM}}$ based on Method of Moment technology is used to analyze and optimize the prototype bandpass filter. The proposed technique was verified practically to validate the design methodology. The experimental results of the prototype circuit are presented and a good agreement was obtained comparing with the simulation results. The dimensions of the proposed filter are $32 \times 24 \mathrm{~mm}^{2}$. The filter's characteristics and compact size make it suitable for wireless communication systems.
\end{abstract}

Keywords: Asymmetric Resonator, Bandpass Filter, Microstrip, Spurious Suppression, Ultra-Wide Stopband

\section{Introduction}

Bandpass filters with high selectivity and excellent out-of-band rejection are essential components in the design of transmitter and receiver employed in microwave wireless communication systems. In particular, the wide stopband bandpass filters are usually utilized in association with the nonlinear components to eliminate the undesired interference or noise in the stopband. It is well known that filters composed of uniform distributed-element resonators, e.g., coupled line filters, open-loop resonator filters suffer from the spurious harmonics at multiples of the fundamental resonance frequency due to higher order resonance inherent in such structures Lin et al. (2006).

Several methods have been proposed to shift the spurious responses higher or to suppress the unwanted passbands. It has been shown suppression of spurious signals can be achieved in the traditional parallelcoupled microstrip filters by over-coupling the end stages Kuo et al. (2003). In another technique a low- pass filter is cascaded with the bandpass filter to eliminate spurious harmonics Chang and Tam (2005). However, the use of an additional low-pass filter degrades the insertion-loss and increases the size of the filter. Harmonic suppression can also be achieved by employing cross-coupling as described in Tu and Chang (2005), where split-ring resonator or open stub and spur line are cross-coupled.

Harmonic suppression over a very wide band was achieved with the assistance of Triangular shaped capacitive loaded at the open-end of the open-loop resonators that incorporate "L" shaped slots and strategically locating open-end stubs within the resonators Virdee et al. (2010). Ultra-compact two-stage microstrip bandpass filter based on twist-modified asymmetric Split-Ring Resonators (SRRs) Tang and Hsu (2008). This kind of filter can achieve three transmission poles within the passband and four transmission zeros below and above the passband. However, the transmission zero are very close to passband. 
Fragment-loaded open-loop resonators in Zhao et al. (2014) shows quite compact dimensional size and well-filtering performance. Moreover, with this structure the spurious passband suppression of the microstrip bandpass filter can be controlled. However, this type of filter is of a complex configuration and there is a trade-off between reducing its size and improving its performance.

Stepped Impedance Resonators (SIR) can be used to shift spurii to a higher frequency by simply modifying the transmission line's width. Other approaches to suppress the higher order harmonics include the minimization of the difference between the even- and odd-mode velocities in coupled structures by equalizing the modal electrical lengths of coupled microstrip lines. This can be achieved by incorporating a slot in the ground-plane. This allows tuning of the even and odd mode phase velocities Kim and Chang (2013) and Kuo et al. (2017). But the process of fabrication of such a structure is complicated and expensive. Thus, the techniques described above are not an effective way to eliminate the spurious responses.

Other examples of harmonic suppression filtering using a combination of techniques have been demonstrated recently by Huang (2015), Tang et al. (2017), Sen and Moyra (2019) and Yang et al. (2019).

In this paper, we propose a microstrip bandpass filter structure which comprises asymmetric resonators interfaced between an inter-digital capacitor Farhat and Virdee (2018). The resulting structure provides an excellent higher order harmonic rejection. The design approach proposed offers high selectivity, excellent insertion-return loss performance across the passband and compact size. This is possible by optimizing parameters of just the inter-digital capacitor.

\section{Filter Design}

Filters with the asymmetric structure generally generate fewer harmonic resonances than their counterpart, e.g. half-wavelength transmission line symmetric structures. Therefore, two asymmetric resonators connected through an inter-digital capacitor, as illustrated in Fig. 1, is proposed here to enhance harmonic rejection generated by microwave filters in order to realize a wide stopband. By changing the parameters of the inter-digital capacitor, we can adjust the magnitude of the coupling between the two resonators that leads to the higher out-of-band rejection and easily tuning the passband.

Analysis was carried out on the effect of interdigital capacitor finger width WS and the separation between fingers $G$ on the center frequency and the bandwidth of the proposed filter. As shown in Fig. 2, as WS increases the center frequency and the bandwidth decrease and this leads for easily tuning the resonant frequency. Where, $\mathrm{G}$ as shown in Fig. 3 has more effect of adjusting the magnitude of the coupling between the two resonators and it leads to tunable bandwidth.

The proposed bandpass filter has been designed for a Centre frequency of $2.66 \mathrm{GHz}$. This was achieved with the following parameter dimensions: $\mathrm{W}=1.7$ $\mathrm{mm}, \mathrm{W} 1=5 \mathrm{~mm}, \mathrm{~W} 2=2.9 \mathrm{~mm}, \mathrm{~L} 1=12 \mathrm{~mm}, \mathrm{~L} 2=$ $5.1 \mathrm{~mm}, \mathrm{~L} 3=7.2 \mathrm{~mm}$ and $\mathrm{G} 1=1.7 \mathrm{~mm} . \mathrm{G}=0.7 \mathrm{~mm}$ and $\mathrm{WS}=0.7 \mathrm{~mm}$.

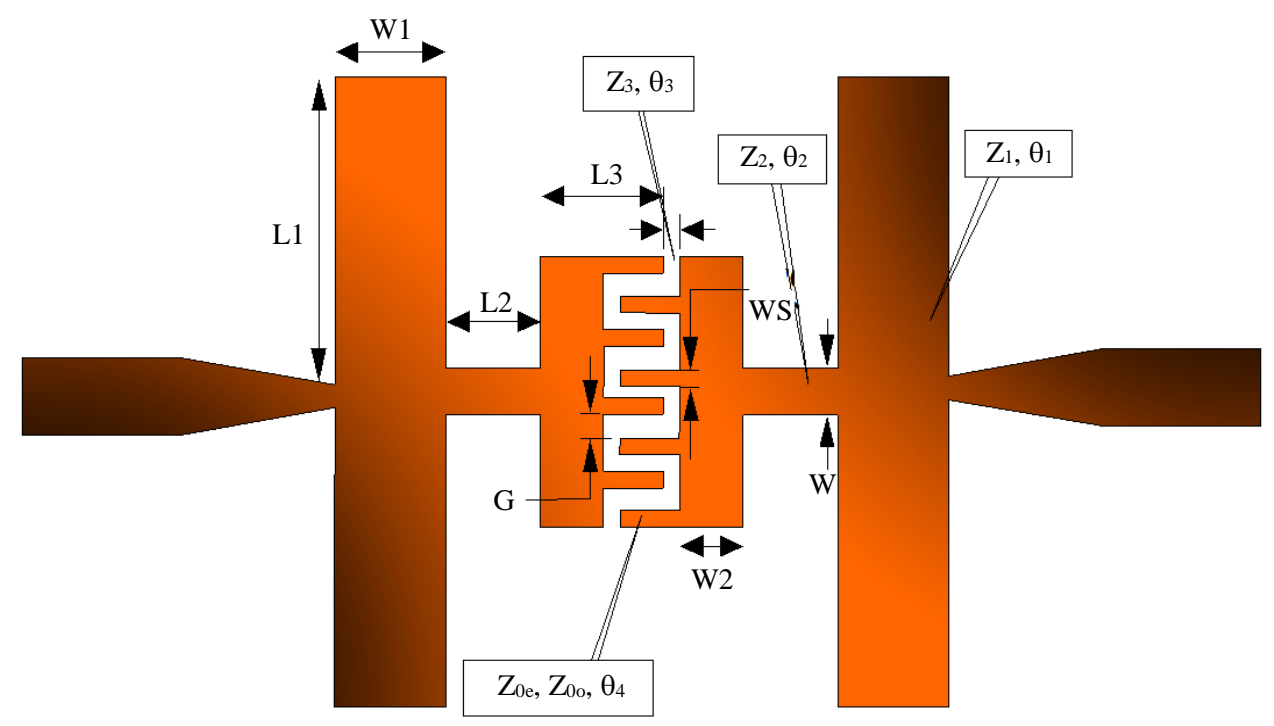

Fig. 1: Schematic diagram of the new proposed filter 


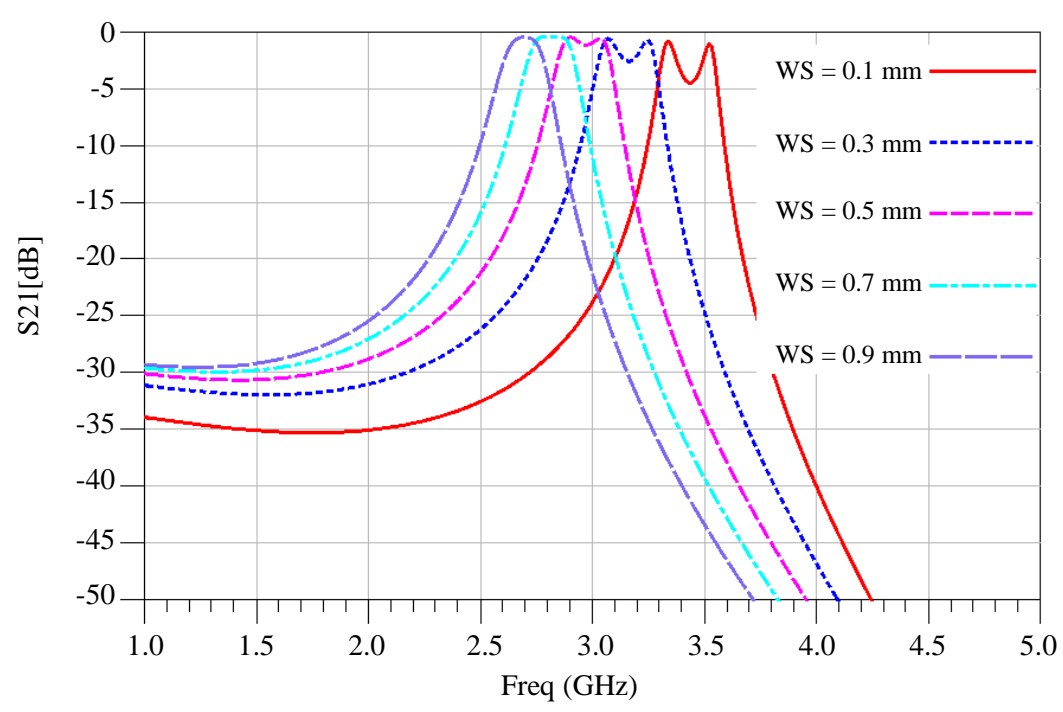

Fig. 2: Relationship between the inter-digital capacitor finger width and normalized resonance frequency

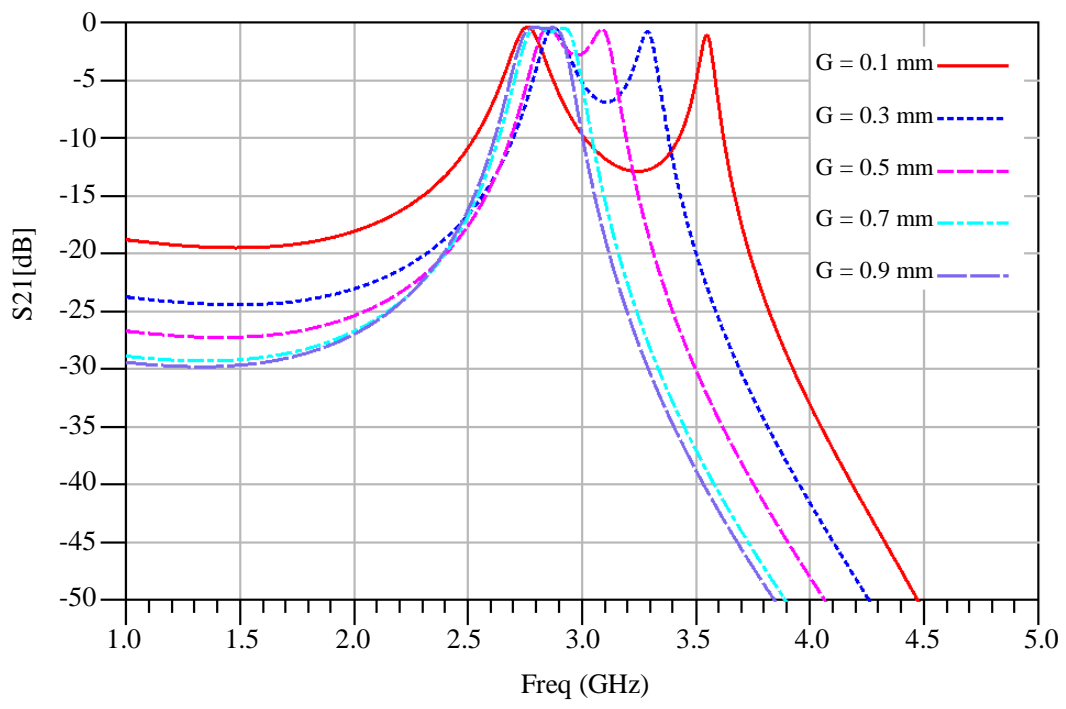

Fig. 3: Frequency response for different separation between inter-digital capacitor fingers width

\section{Synthesis of the Passband Filter}

The proposed bandpass filter transformed into the equivalent circuit shown in Fig. 4 with the immittance inverter. The transformed admittance inverter $J_{12}$ and $J_{23}$ of transmission lines $Z_{2}$ and $Z_{3}$ and the even and oddmode line admittances $Y_{\mathrm{o} e}$ and $Y_{\mathrm{o} o}$ can be derived for a lossless case as Matthael et al. (1980):

$J_{12}=Y_{2} \csc \theta_{2}$

$J_{23}=Y_{3} \csc \theta_{3}$

$\frac{Y_{o e}}{Y_{4}}=1-\frac{J_{34}}{Y_{4}} \cot \theta_{4}$
$\frac{Y_{O_{0}}}{Y_{4}}=1+\frac{J_{34}}{Y_{4}} \cot \theta_{4}$

By matching with the generalized bandpass filter, the parameters of susceptance (B) and susceptance slope (b) can be than expressed as following Matthael et al. (1980):

$B_{1}=Y_{1} \tan \theta_{1}-Y_{2} \cot \theta_{2}$

$B_{2}=-Y_{2} \tan \theta_{2}-Y_{3} \cot \theta_{3}$

$B_{3}=Y_{4} \tan \theta_{4}-Y_{3} \cot \theta_{3}$ 


$$
\begin{aligned}
& b_{1}=\left.\frac{\omega_{o}}{2} \frac{\delta B_{1}}{\delta \omega}\right|_{\omega=\omega_{o}}=\frac{1}{2}\left[Y_{1} \theta_{1} \sec ^{2} \theta_{1}+Y_{2} \theta_{2} \csc ^{2} \theta_{2}\right] \\
& b_{1}=\frac{R_{s} g_{0} g_{1} J_{01}^{2}}{\Delta} \\
& b_{2}=\left.\frac{\omega_{o}}{2} \frac{\delta B_{2}}{\delta \omega}\right|_{\omega=\omega_{o}}=\frac{1}{2}\left[Y_{2} \theta_{2} \csc ^{2} \theta_{2}+Y_{3} \theta_{3} \csc ^{2} \theta_{3}\right] \\
& J_{12}=\Delta \sqrt{\frac{b_{1} b_{2}}{g_{1} g_{2}}}=\frac{1}{2}\left[Y_{4} \theta_{4} \sec ^{2} \theta_{4}+Y_{3} \theta_{3} \csc ^{2} \theta_{3}\right] \\
& b_{3}=\frac{\omega_{o}}{2} \frac{\delta B_{3}}{\left.\delta \omega\right|_{\omega=\omega_{o}}}=\Delta \sqrt{\frac{b_{2} b_{3}}{g_{2} g_{3}}}
\end{aligned}
$$

where, $g_{i}$ are the elements values, $\omega_{0}$ is angular frequency in center frequency and $\Delta$ is the relative bandwidth.

Therefore,

$$
\begin{aligned}
& Y_{1}=\frac{2 b_{1}-Y_{2} \theta_{2} \csc ^{2} \theta_{2}}{\theta_{1} \sec ^{2} \theta_{1}} \\
& Y_{3}=\frac{2 b_{2}-Y_{2} \theta_{2} \csc ^{2} \theta_{2}}{\theta_{3} \csc ^{2} \theta_{3}} \\
& Y_{4}=\frac{2 b_{3}-Y_{3} \theta_{3} \csc ^{2} \theta_{3}}{\theta_{4} \sec ^{2} \theta_{4}}
\end{aligned}
$$

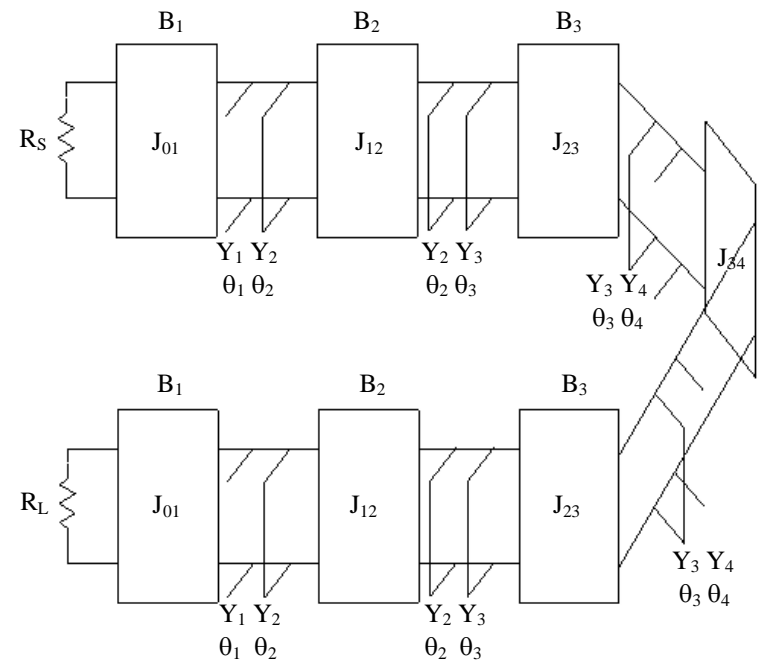

Fig. 4: Equivalent circuit of the proposed microstrip BPF

\section{Asymmetric Resonant Structure}

The proposed bandpass filter in Fig. 1 is an asymmetric structure. Hence, the resonant condition for this step impedance resonator (SIR) can be expressed as Tang and Hsu (2008):

$R_{1}=\tan \theta_{1} \tan \theta_{2}$

$R_{1}\left(R_{3}-\tan \theta_{3} \tan \theta_{4}\right)=\tan \theta_{2}\left(R_{3} \tan \theta_{3}+\tan \theta_{4}\right)$

Where:

$$
\begin{aligned}
& R_{1}=Z_{1} / Z_{2}, R_{2}=Z_{3} / Z_{2} \text { and } R_{3}=Z_{4} / Z_{3} \\
& Y_{1}=\frac{Y_{2}}{\tan \theta_{1} \tan \theta_{2}} \\
& Y_{4}=\frac{Y_{2}-Y_{3} \tan \theta_{2} \tan \theta_{3}}{\tan \theta_{4}\left(\frac{Y_{2}}{Y_{3}} \tan \theta_{3}+\tan \theta_{2}\right)}
\end{aligned}
$$

Using the above expressions, the electrical length $\theta_{1}, \theta_{2}$ and $\theta_{3}$, the impedance $Z_{1}, Z_{3}, Z_{\mathrm{o} e}$ and $Z_{\mathrm{oo}}$ obtained are: $53.18^{\circ}, 26.17^{\circ}, 17.73^{\circ}, 29.98^{\circ}, 45 \Omega$, $160.7 \Omega$ and $157 \Omega$ respectively. Furthermore, the optimized values for a desired response the impedance $\mathrm{Z}_{2}$ and electrical length $\theta_{4}$ are $63.84 \Omega$ and $21.91^{\circ}$, respectively.

\section{Simulation, Fabrication and Measurement}

To demonstrate the practical feasibility of the proposed concept, in Fig. 1 it was fabricated using standard photolithographic circuit board techniques on a dielectric substrate with dielectric constant $\left(\varepsilon_{r}\right)$ of 2.17, thickness $(h)$ of $0.794 \mathrm{~mm}$ and loss tangent $(\tan \delta)$ of 0.0009 . In order to make it simpler and easier to fabricate, inverters $\mathrm{J}_{01}$ are set to $0.02\left(1 / Y_{0}\right)$ which equates to the system impedance of $50 \Omega$. Therefore, a transmission line of $50 \Omega$ can be directly connected to the input and output ports in this bandpass filter.

Fig. 5 shows a photograph of the filter, Fig. 6 shows the simulation results and Fig. 7 its measured performance. The filter has high out-of-band rejection (> $29 \mathrm{~dB}$ ), less than $0.6 \mathrm{~dB}$ of insertion-loss, up to 5.5 $\mathrm{GHz}$ spurii free and about $15 \mathrm{~dB}$ of return-loss. Comparing the simulation with the measured result shows excellent agreement. 


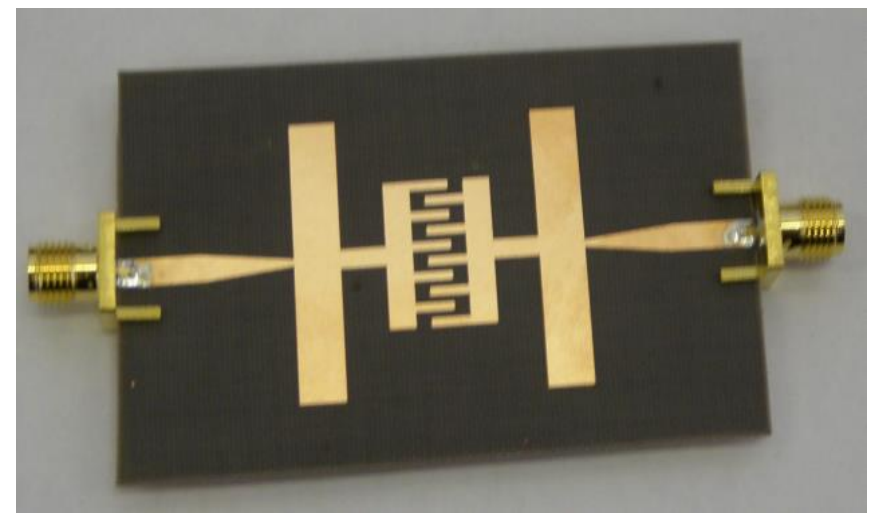

Fig. 5: Photograph of the fabricated proposed filter

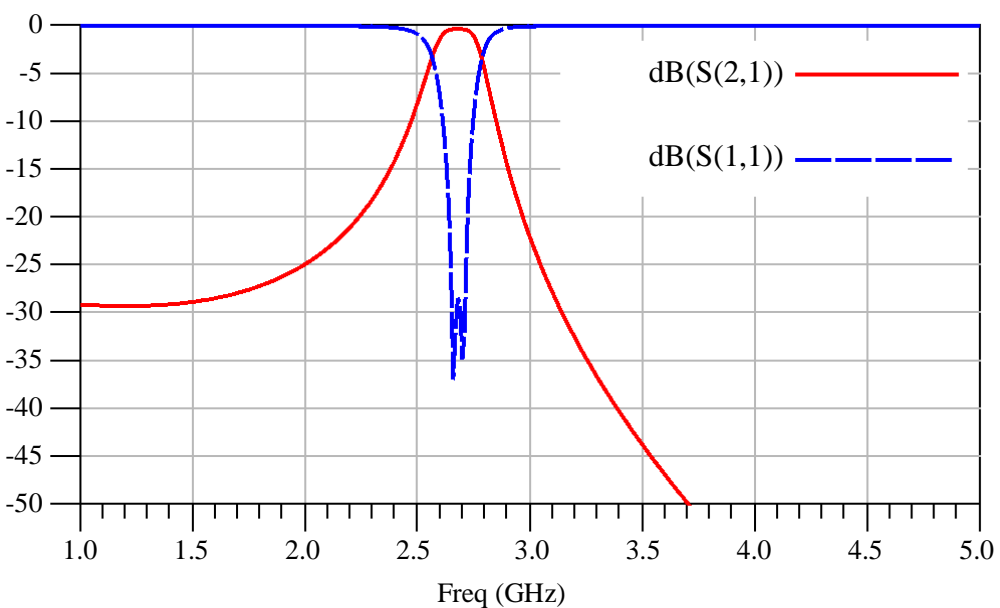

Fig. 6: The simulation results of the proposed filter

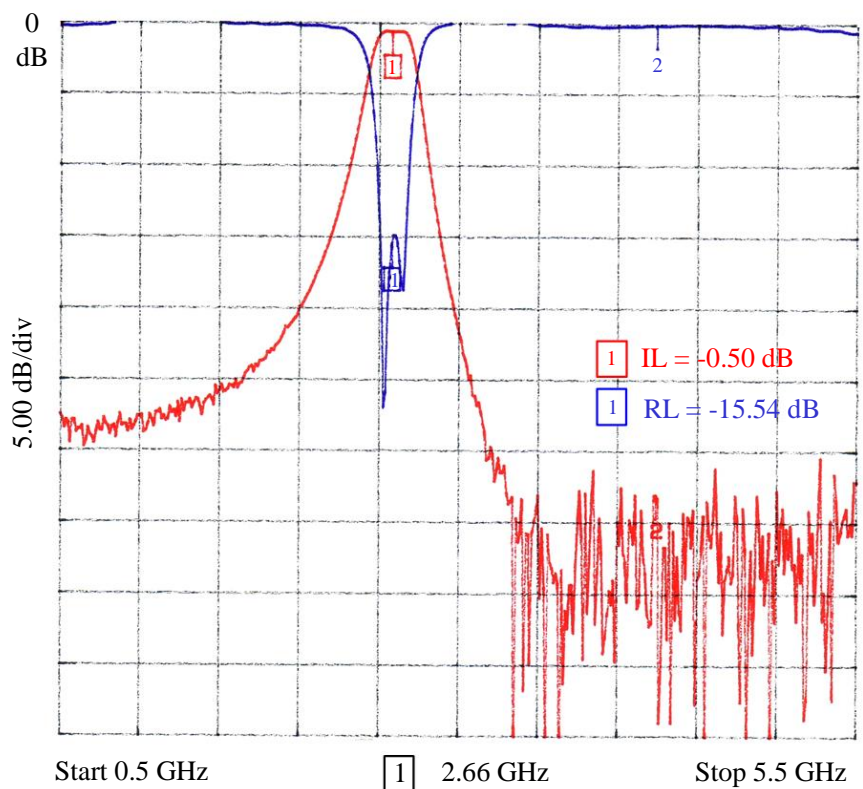

Fig. 7: The measured result of the proposed filter 


\section{Conclusion}

A new configuration comprised of asymmetric structure and inter-digital capacitor has been presented to design a high performance bandpass filter with high outof-band rejection and wide stop band. The presented technique allows control of the centre frequency and the fractional bandwidth simple by varying the inter-digital capacitor parameters. Also the design methodology of the proposed filter is simple and easy to fabricate. Comparing to those reported in references the measured and simulation results of the proposed bandpass filter shows good improvement over passband insertion- loss and return-loss. The proposed filter exhibits a wide stopband, high selectivity that is required in modern communication systems for suppressing undesired interferences from other wireless systems.

\section{Author's Contributions}

Mohamad Farhat and Bal Virdee: Conducted the research and participated in writing the manuscript.

Nader Ghareeb: Performed the fine tuning simulation and participated in writing the manuscript.

\section{Ethics}

This article is an original research paper. There are no ethical issues that may arise after the publication of this manuscript.

\section{References}

Chang, K.F. and K.W. Tam, 2005. Miniaturized crosscoupled filter with second and third spurious responses suppression. IEEE Microw. Wireless Compon. Lett., 15: 122-124.

DOI: 10.1109/LMWC.2004.842857

Farhat, M. and B.S. Virdee 2018. Microstrip Bandpass filter with wide stopband and high out-of-band rejection based on inter-digital capacitor. Int. J. Electron. Commun. Eng.

Huang, F., 2015. Suppression of harmonics in microstrip filters using a combination of techniques. IEEE Trans. Microwave Theory Techniques, 63: 3453-3461. DOI: 10.1109/TMTT.2015.2461616

Kim, C.H. and K. Chang, 2013. Wide-stopband bandpass filters using asymmetric steppedimpedance resonators. IEEE Microw. Wireless Compon. Lett., 23: 69-71. DOI: 10.1109/LMWC.2012.2236885

Kuo, J.T., S.P. Chen and M. Jiang, 2003 Parallel-coupled microstrip filters with over-coupled end stages for suppression of spurious responses. IEEE Microw. Wireless Compon. Lett., 13: 440-442.

DOI: 10.1109/LMWC.2003.818531
Kuo, J.T., Y.T. Lu and H.M. Lin, 2017. Planar dualmode dual-band dual-ring resonator bandpass filter. Proceedings of the IEEE MTT-S International Conference on Numerical Electromagnetic and Multiphysics Modeling and Optimization for RF, Microwave and Terahertz Applications, May 17-19, IEEE Xplore Press, Seville, Spain pp: 4-6.

DOI: 10.1109/NEMO.2017.7964168

Lin, S.C., P.H. Deng, Y.S. Lin, C.H. Wan and C.H. Chen, 2006. Wide-stopband microstrip bandpass filters using dissimilar quarter-wavelength steppedimpedance resonators. IEEE Trans. Microwave Theory Techniques, 54: 1011-1018. DOI: 10.1109/TMTT.2005.864139

Matthael, G., L. Young and E.M.T. Jones, 1980. Microwave Filters, Impedance-Matching Network and Coupling Structures. 1st Edn., Artech, Norwood, MA, ISBN-10: 0890060991, pp: 1096.

Sen, S. and T. Moyra, 2019. Compact microstrip lowpass filtering power divider with wide harmonic suppression. IET Microwaves Antennas Propagat., 19: 2026-2031. DOI: 10.1049/iet-map.2019.0222

Tang, C., X. Lin, W. Liu and Y. Fan, 2017. Wide stopband bandpass filters based on quarterwavelength resonators. Microwaves Antennas Propagat. IET, 11: 1379-1388.

Tang, C.W. and Y.K. Hsu, 2008. Design of a wide stopband microstrip bandpass filter with asymmetric resonators. IEEE Microw. Wireless Compon. Lett., 18: 91-93. DOI: 10.1109/LMWC.2007.915034

Tu, W.H. and K. Chang, 2005. Compact microstrip bandstop filter using open stub and spurline. IEEE Microw. Wireless Compon. Lett., 15: 268-270. DOI: 10.1109/LMWC.2005.845739

Virdee, B.S., M. Farhat and M.N. Moghaddasi, 2010. Ultra-wideband spurii-free quasi-elliptic function filter. Proceedings of the Asia-Pacific Microwave Conference, Dec. 7-10, IEEE Xplore Press, Yokohama, pp: 1201-1204.

Yang, X., H. Luyen, S. Xu and N. Behdad, 2019. Design method for low-profile, harmonic-suppressed filterantennas using miniaturized-element frequency selective surfaces. IEEE Antennas Wireless Propagat. Lett., 18: 427-431. DOI: $10.1109 /$ LAWP.2019.2893245

Zhao, Q., G. Wang and D. Ding, 2014.Compact microstrip bandpass filter with fragment-loaded resonators. Microw. Opt. Technol. Lett., 56: 2896-2899. DOI: $10.1002 / \mathrm{mop} .28726$ 